\title{
Bringing the NDE Home
}

To the Editor:

I recently read a copy of Barbara Harris and Lionel Bascom's book Full Circle, which pointed out for me the problems involved in making a transition from the near-death experience (NDE) back to the less luminous world. Harris and Bascom phrased the question in something of the form: "If the reality of life as discovered in the NDE is light and love, why are our lives so often in turmoil?"

The answer is, to use an analogy from earthly journeys, that visiting Paris does not make a person a Parisian. To be a Parisian a person has to take up residence in Paris. For the NDEr this involves making the perceptions during the NDE the core of your belief system, your intellectual home.

The first step in this process is accepting the experience and accept- 
ing the reality of the experience. To my mind, if a theologian or a clinician counseled an NDEr that the NDE is a useful imaging, that would set up a tension making the experience more difficult to integrate. Once you have accepted that you have experienced what for generations has been called "the divine," or at least a significantly different dimension of reality, the process of integrating the experience can begin.

A useful mental technique for establishing the NDE perceptions as the core of a person's belief system is what I call the "TV meditation," the method of spiritual advancement achieved through watching television. While watching TV a person's attention will often be caught up by the action on the screen; consciousness will be in the screen action. While breathing normally and in whatever position is comfortable, the spiritual aspirant should shift his or her consciousness back so that he or she is aware of the action on the screen and of the television set and of the room around the television. This discipline can be carried out whenever the spiritual aspirant thinks of doing it; it can be done even while consuming potato chips.

With practice the aspirant will be able to carry over this shifting of consciousness to the non-TV world. In the non-TV world you can shift consciousness between the action you are involved in and how the same action would look in the light of the NDE. When your kids, your boss, or the person in the next car start getting to you, step back mentally and try seeing these people not as out to make you miserable, but as trying to act in the light but being confused about what they're doing, as Harris and Bascom suggested. When the refocusing becomes second nature, the turmoil decreases and is replaced by a compassion for their confused efforts.

The TV meditation technique does not work overnight, but neither does it require living in a cave. With a moderate amount of effort, it probably gets results faster than eating brown rice for long periods of time.

For the adventurous, this centering on the NDE can be taken some steps further, allowing the perceptions gained during the NDE to take over more and more of one's consciousness. This process within the framework of the Roman Catholic tradition was described in a turn-ofthe-century book, The Graces of Interior Prayer (Poulain, n.d.), which details how the aspirant moves from the prayer of quiet, in which one spends longer or shorter moments in the refocused state of light, to the prayer of union, in which one becomes an active part of the light.

There are pitfalls to this more rigorous practice. One potential haz- 
ard is that of being overwhelmed by the experience and losing control of the outward life. Another is turning inward and living only for the experience with a lessening of interest in the outer life. Both of these may be overcome by bringing more light into the material world.

Psychic abilities are a more sophisticated pitfall and their varieties have been described by Patanjali in his Yoga Sutras. In addition to outof-body experiences, thought transference, and bodily sympathy or feeling another's feelings at a distance, levitation and the ability to see through solid objects are said to appear at times to distract the aspirant. Because these abilities are so novel, some aspirants stop their refocusing practices at this point; others will keep at it until light and life are one.

Perhaps other readers can add details to this theme.

\section{References}

Harris, B., and Bascom, L.C. (1990). Full Circle: The near-death experience and beyond. New York, NY: Pocket Books.

Poulain, A. (n.d.). The graces of interior prayer (Des graces d'oraison) (L.L.Y. Smith, Trans.). St. Louis, MO: Herder.

Oliver Nichelson 333 North 760 East American Fork, UT 84003 